\title{
Infrared Remote Control Intelligent Digital Calendar Design
}

\author{
Tang Lili, a , Huang Wei ${ }^{2, b}$ \\ ${ }^{1}$ Department of Computer \& Information Engineering, Zhixing College of Hubei University, Wuhan, \\ 430011, China \\ ${ }^{2}$ System Design Institute of Hubei Aerospace Technology Academy, Wuhan, 430040, China \\ aemail: toney2001@126.com, bemail:jijunhuang@126.com
}

Keywords: Digital calendar; Respiratory display; Infrared remote control

\begin{abstract}
This article comprehensively and systematically introduces the design of infrared remote control breathing show intelligent electronic calendar. Design using microcontroller AT89S52 as the core chip. The system structure consists of AT89S52, DS1302 time chip, DS18B20 digital temperature sensor chip and other components. The whole design includes hardware circuit design and software design. Hardware circuit design includes I/O assignment, select the external device components, data exchange circuit design, and software design is mainly for data communications and logic control of each unit.
\end{abstract}

\section{Introduction}

Now, application microprocessor technology, so to further improve the accuracy of timing products, but also includes a variety of daily information, not just the original monotonous time function. Development from the existing traditional analog timekeeping manner more familiar everyday LED digital display or LCD digital display, intuitive and clear, and with automatic date, week, temperature, and other everyday affiliated display of information, therefore, the emergence of electronic calendar chronograph watch industry has brought leap of progress.

Infrared remote control intelligent digital calendar uses a digital chip control internal data operation, infrared remote control with LED luminous digital display date, time, day, solar terms countdown, as well as temperature and other daily information, it is a blend of a number of advanced electronic technology, modern digital timing products.

\section{Working principle}

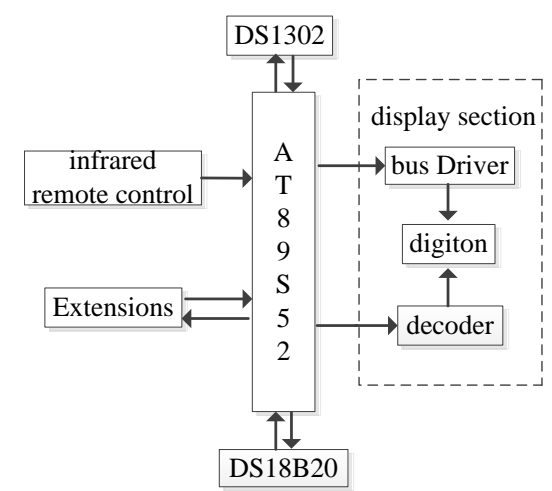

Fig.1. Hardware system block diagram

Time information is written to the chip DS1302 time, read out and displayed on the digital conversion by AT89S52. Also writing instruction to the DS18B20, reading temperature information and displayed on the digital tube after the conversion. When an interrupt request using infrared control, executes the interrupt program, decodes the infrared signal and adjusts time, alarm [1], etc.

\section{AT89S52 Chip Introduction}

AT89S52 is American Atmel's high performance CMOS8 bit microcontrollers, having a wealth of on-chip resources, including an $8 \mathrm{~K}$ in-system programmable Flash memory, interrupt sources, 
watchdog timer module, three 16-bit timer / counters [2] [3], etc.

\section{Main Modules Introduction}

\section{DS1302 Introduction}

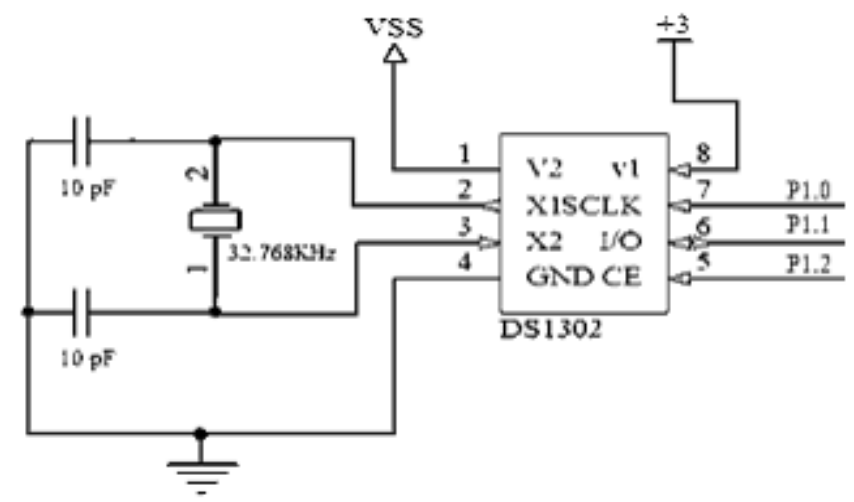

Fig.2. DS1302 module circuit diagram

The DS1302 consists of two power supply, VSS main power supply of 5V, 3V standby power. $32.768 \mathrm{khz}$ crystal provides clock frequency. DS1302 through two control port and an I/O port to communicate with the microcontroller. It is primarily responsible for storing the timing and alarm clock, IR command information.

\section{DS18B20 Introduction}

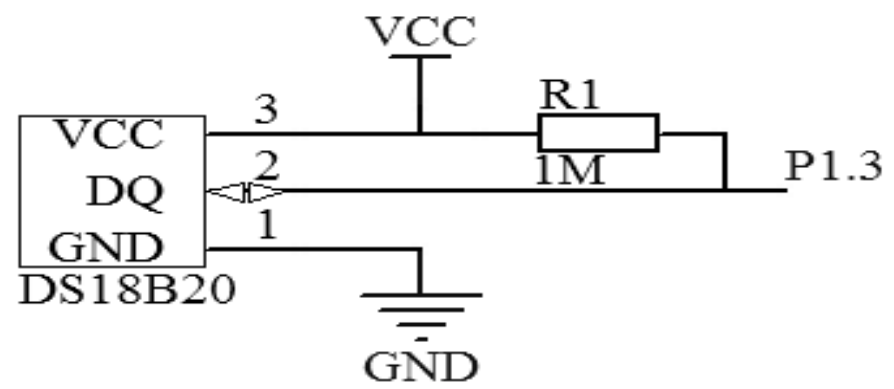

Fig.3. DS18B20 circuit diagram

The DS18B20 module consists of a 5V supply, DQ for I /O port, through it reading data from the microcontroller or sending data to the microcontroller. Since DQ is an open drain, which can not produce a high level. Therefore, pressing the $1 \mathrm{M}$ pull-up resistor between DQ and VCC. It is mainly responsible to measure the temperature and convert into digital signal identified by microcontroller.

\section{Display Introduction}

The display module composed by the 16 highlight common cathode LED, It is driven using 74LS245, 4-16 line decoder consisted by two 74LS138 controls 16 PNP transistor and its dynamic scan. During the scanning process can adjust brightness by adjusting for digital control of each transistor conduction time [4]. On-time is shorter, low brightness, the brighter and vice versa. But the single digital control conduction time is too long will cause the display discontinuous, reaching effect not displayed simultaneously. 


\section{IR receiver Introduction}

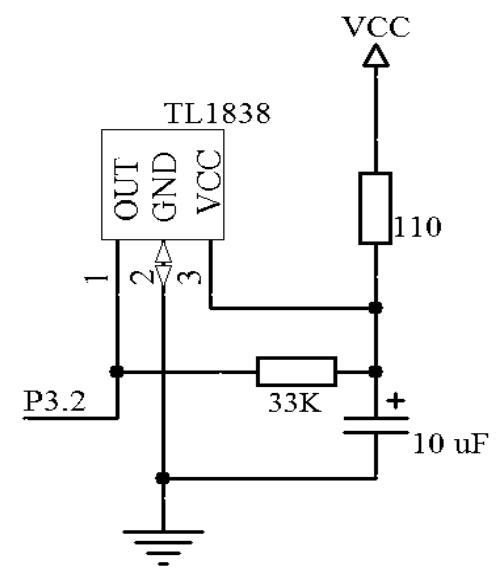

Fig.4. IR receiver module circuit diagram

The IR receiver module consists of TL1838 integrated infrared receiver composition, receiving modulated infrared signal amplification, demodulation, and output the inverted level signal. Since the gain of the external receiving head is very large, it is easy to cause interference, so the RC filter is required on the receiving head of the power supply. The output is open-drain, coupled with its 33K pull-up resistor.

\section{Infrared remote control system}

The universal infrared remote control system is composed of two parts, which are transmitted and received, and the application of the integrated circuit chip is used to control the operation. The transmitting part comprises a keyboard matrix, an encoding modulation, a LED infrared transmitter; the receiving part comprises a light, an electric conversion amplifier, a demodulation and decoding circuit [5].

\section{Respiratory display}

There are two ways of dynamic and static in the digital tube display. Static is at the same time to light up all the digital tube, such a way to compare the use of single-chip I/O resources, especially the number of digital tube. Dynamic display can use less I/O resources. Dynamic display refers to each digital tube will be lit, called scanning. When the scanning speed is relatively fast, the human eye sees as the same as the light.

In the actual process, in order to make the maximum brightness of the digital tube, we will put a digital tube lights up after 100 to 200us. At this point you can control the length of the delay time to control the brightness of the digital tube. In the scanning process, the delay time can be changed from short to long and to short, then the digital tube can be changed from dark to bright and to dark, that is the effect of the call and suction. 


\section{Main software design}

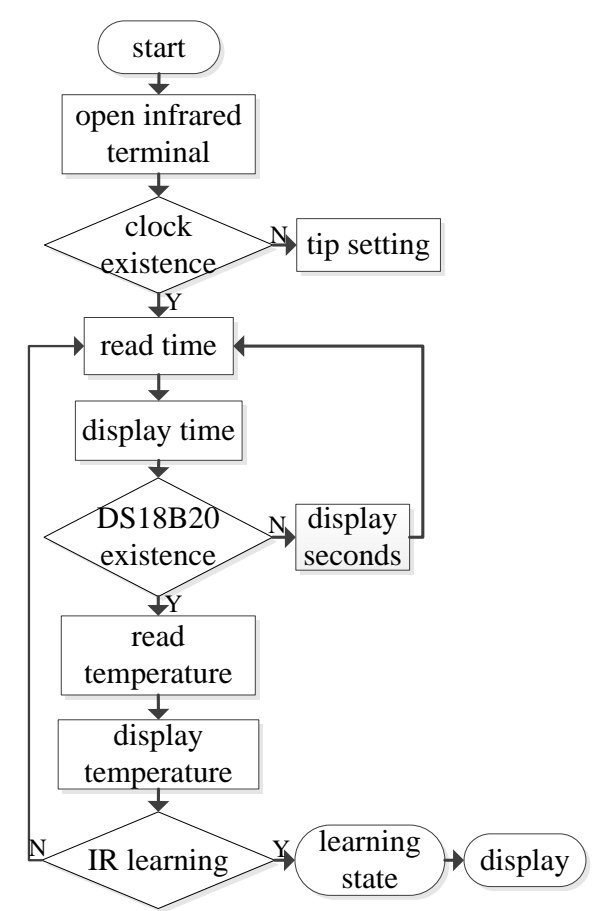

Fig.5. Main software design flow chart

Software design is a part of the design of the most difficult, it needs to put all in one, because of the function of the design to the each module integrated together to coordinate their efforts and need to set up multiple interrupt, to control all kinds of interruption of the opening time and closing time, software design including infrared decoding, DS1302, DS18B20 read and write, dynamic digital tube display.

After the system is started, the DS1302 is detected with time data. And then store, display the time temperature (no temperature sensor display seconds). It executes the program when there is a corresponding interrupt instruction.

\section{Conclusion}

This paper mainly discusses the common chip and single-chip connection and their operation. The 8 bit microprocessor AT89S52 is used as the control core, and the full use of various resources, the use of infrared remote control technology, through the digital tube dynamic display. The design of infrared remote control breathing show intelligent digital calendar has the advantages of simple structure, convenient operation, stable and reliable, in the actual use of the process to achieve a certain timing, temperature measurement accuracy, and can meet the requirement of daily life.

\section{Acknowledgement}

In this paper, the research was sponsored by the Science and Technology Research Program of 2015 Hubei Provincial Education Department (Project No. B2015514)

\section{References}

[1] Kang Huaguang. Part of the simulation of electronic technology basis [M]. Beijing: Higher Education Press, 2008.

[2] Qiushi Technology. 8051 Series MCU C program design [M]. Beijing: People's Posts and Telecommunications Publishing House, 2006. 
[3] He Jianguo, Zheng Yan, Xue Yanxia. Application of single chip microcomputer in electronic circuit design [M]. Beijing: Tsinghua University Press, 2006.

[4] Liu Bihu, Shen Jianguo. Digital logic circuits [M]. Beijing: Science Press, 1999.

[5] David Cook. Robot Building for Beginners [M].New York: Apress,2011. 